\title{
Healthcare Misinformation Detection and Fact-Checking: A Novel Approach
}

\author{
Yashoda Barve ${ }^{1}$, Jatinderkumar R. Saini ${ }^{2 *}$ \\ Suryadatta College of Management Information Research and Technology, Pune, India ${ }^{1}$ \\ Symbiosis Institute of Computer Studies and Research, Symbiosis International (Deemed University), Pune, India ${ }^{2}$
}

\begin{abstract}
Information gets spread rapidly in the world of the internet. The internet has become the first choice of people for medication tips related to their health problems. However, this ever-growing usage of the internet has also led to the spread of misinformation. The misinformation in healthcare has severe effects on the life of people, thus efforts are required to detect the misinformation as well as fact-check the information before using it. In this paper, the authors proposed a model to detect and factcheck the misinformation in the healthcare domain. The model extracts the healthcare-related URLs from the web, preprocesses it, computes Term-Frequency, extracts sentimental and grammatical features to detect misinformation, and computes distance measures viz. Euclidean, Jaccard, and Cosine similarity to fact-check the URLs as True or False based on the manually generated dataset with expert's opinions. The model was evaluated using five state-of-the-art machine learning classifiers Logistic Regression, Support Vector Machine, Naïve Bayes, Decision Tree, and Random forest. The experimental results showed that the sentimental features are crucial while detecting misinformation as more negative words are found in URLs containing misinformation compared to the URLs having true information. It was observed that Naïve Bayes outperformed all other models in terms of accuracy showing $98.7 \%$ accuracy whereas the decision tree classifier showed less accuracy compared to all other models showing an accuracy of $\mathbf{9 2 . 8 8 \%}$. Also, the Jaccard Distance measure was found to be the best distance measure algorithm in terms of accuracy compared to Euclidean distance and Cosine similarity measures.
\end{abstract}

Keywords-Misinformation detection; sentiment analysis; document similarity; fact-check; healthcare

\section{INTRODUCTION}

Online social media and the web as a whole have become the spring of information to users all around the world. Due to its convenience, feasibility, unrestricted access, and reasonable cost the internet have become popular amongst the community [1], [2]. The people read, share, write, and view the articles, blogs, news, videos, audios, etc., all over the internet. The rate of sharing articles, blogs, news, etc., has been accelerated dramatically. However, the users not only share immaculate information but also try to spread wrong or incorrect information either knowingly or unknowingly in a moment. This widespread misinformation has relentless consequences on individuals, commercial, health, government, and all other facets of society. The ramification of the misinformation is catastrophic and may lead to extermination. For example, the political disinformation spread during the 2016 USA presidential elections led to public shootings. These enduring consequences of misinformation contribute towards ferocious conflicts that are preventable otherwise [3], [4].

The internet has become the most popular and the first choice of the public to investigate health problems. However, people get misinformed with wrongly populated content. A famous and perfect example is the misconception among the public about the measles, mumps, and rubella (MMS) vaccine causing autism. Health misinformation is defined as "A healthrelated claim of fact that is currently false due to a lack of scientific evidence" [5]. The promulgated experiences of people over the internet or articles were written about certain diseases without knowing or verifying the fact or having a lack of evidence can cause health ruination of readers and thus can lead to complete desolation [6], [7]. The misinformation related to health can have hazardous effects on people's life directly, thus detecting misinformation in healthcare is a need of time [8]-[10].

Misinformation detection has become the topic of interest amongst researchers in the literature. The researchers have studied different types of false information. The first category is termed misinformation, which is the inaccurate or incorrect information that is confirmed with existing evidence [11]. The other categories include the fake news [12], [13], rumor [6], satire news [14], hoaxes [15], misinformation [16], [17], disinformation [18] and opinion spam [19]. To detect each of these categories of false information the authors have used several features like sentiment analysis, user-specific features, syntactical features, grammatical features, image or message specific features, etc. Also, there are readily available datasets for false information detection in various domains viz. politics, news, business, and healthcare. Few examples of these datasets are LIAR, FakeNewsNet, BSDetector, etc. With the help of features and datasets, machine learning and deep learning techniques are applied to detect false information [11]. However, detecting misinformation is an exhaustive task. This is due to two main reasons: first, is the availability of dataset in a certain domain and second is fact-checking of the data [11], [20], [21]. It is difficult to get the benchmark and gold-standard datasets in a specific domain. Also, manual fact-checking of data is time-consuming, requires expert guidance, and involves laborious tasks. Thus, automatic fact-checking of data is a need of time to endure with the speed of the newly arriving and changing data.

Document Similarity is a measure of the distance between the two documents (DS). There are several distance measures available in the literature to compute the similarity between the

\footnotetext{
*Corresponding Author.
} 
documents like Euclidian Distance, Cosine Similarity, Jaccard Distance, etc. The concept of document similarity can be used to fact-check the information with the existing verified documents and thus can help to detect misinformation. Document Similarity is a measure of the distance between the two documents (DS). There are several distance measures available in the literature to compute the similarity between the documents like Euclidian Distance, Cosine Similarity, Jaccard Distance, etc. The concept of document similarity can be used to fact-check the information with the existing verified documents and thus can help to detect misinformation.

Sentiment Analysis (SA) techniques to detect the polarity of data into positive, negative, and neutral have been widely used in the literature to detect misinformation, fake news, rumors, etc. The process of knowing the opinion of the people about the products, services, movie reviews, etc. can be easily captured using sentiment analysis [20], [22]-[26]. The literature related to misinformation detection or finding the credibility of information using sentiment analysis has marked that the articles or blogs containing more positive words are tend to be spreading true information while the articles having negative information contain more negative information [27], [28].

Thus, to detect misinformation and perform fact-checking automatically the authors have proposed a hybrid approach of sentiment analysis and document similarity. In this research paper, the authors have created a sentiment-based Bag-ofWords (BoW) as a dataset related to the healthcare domain. Further, features like sentiment analysis, grammatical and lexical features are used to detect misinformation and document similarity measures viz. Euclidian distance, Cosine similarity, and Jaccard distance are used to perform factchecking.

The remaining sections of the paper are structured as follows: Section II provides the literature survey describing the techniques of using sentiment-based features to detect misinformation in the healthcare domain and also the document similarity-based approaches used to fact-check the documents which could help to detect misinformation in the healthcare domain. Section III describes the proposed model architecture, dataset collection and cleaning process, and methodology used in the proposed model. Section IV discusses the results generated based on the proposed model of a hybrid approach of sentiment analysis and document similarity and section $\mathrm{V}$ describes the conclusion and future enhancements.

\section{RELATED WORK}

\section{A. Sentiment Analysis in Healthcare}

In terms of web articles, the sentiment analysis is an expression that measures the attitude of the author in terms of positive, negative or neutral towards the article topic. Especially, when talked about healthcare-related articles, people like to express and share their opinions about their experiences about the disease which they have suffered from. Therefore the readers get biased towards the opinion of the author and believe the article without verifying the facts or evidence. Due to the rich contents of health information available online, the web has become the first choice of patients or users to know about the cure of disease and related remedies. Thus, understanding the sentiment of the article contents is much needed when it comes to misinformation detection. In the state-of-the-art techniques, the authors have analyzed the moods of cancer patients from tweets. Long Short Term Memory (LSTM) techniques were used to find the sentiments from the tweets [29]. In another research, authors collected 1,000 text comments of medical experts through various medical animation videos of the Youtube repository, and applied sentiment analysis to these comments to enhance the reputation of telemedicine education across the globe [30]. To study the effectiveness or popularity of a medicine, authors have performed sentiment analysis on public reviews using weighted word representation techniques and added linguistic constraints to model the contextually similar words [31]. Also, sentiment analysis techniques were used to detect misinformation in herbal treatments of diabetes in Arabic comments of YouTube videos [32]. The sentiment analysis is widely used in the healthcare sector to understand the sentiment polarity of the text and thus it can act as a major feature for misinformation detection. Table I displays the recent techniques of sentiment analysis in the healthcare domain in comparison with the proposed model techniques.

\section{B. Document Similarity in Healthcare}

Document similarity measures the distance between two documents in a numeric value. The document similarity measures are used to find the similarity between healthcare documents. For example, to detect medical codes of the documents the authors have used an attention mechanism which targets the most informative parts of the documents [33]. In another research, Jaccard distance measure was used to compute the similarity between medical documents using a Non-negative matrix factorization algorithm [34]. In another research, the Term Frequency-Inverse Document Frequency (TF-IDF) of a document is computed and document similarity is measure using cosine similarity, further k-means is used to cluster the documents of similar types. The authors have also used the Unified Medical Language System (UMLS) to extract domain-specific features and select the required features using Principal Component Analysis (PCA). Further, the authors have used expected maximization techniques to cluster the similar documents together [35]. The document similarity is extensively applied in the healthcare domain to group similar documents together. This technique along with sentimental features will be useful for detecting misinformation in the healthcare domain. Table II displays the recent techniques of document similarity in the healthcare domain concerning the proposed model. 


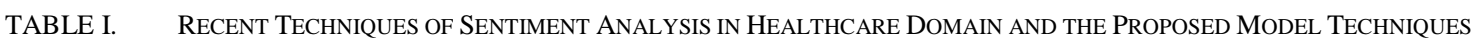

\begin{tabular}{|l|l|l|l|l|l|}
\hline Sr. No. & Reference & Technique & Dataset & Features & Sentiment Classification \\
\hline 1 & {$[29]$} & LSTM & 821,483 public tweets & N-gram, TF-IDF,LDA, PCA & Cancer Tweets \\
\hline 2 & {$[30]$} & $\begin{array}{l}\text { Classifiers used like } \\
\text { SVM, kNN }\end{array}$ & 1,010 comments & Sentiment & Medical Videos \\
\hline 3 & {$[31]$} & $\begin{array}{l}\text { Classifiers used like } \\
\text { SVM, kNN }\end{array}$ & $2,15,063$ patient reviews & TF-IDF & Patient Reviews \\
\hline 4 & Proposed Model & $\begin{array}{l}\text { Classifiers used like } \\
\text { LR, SVM, NB, DT, RF }\end{array}$ & $\begin{array}{l}\mathbf{1 0 0 0} \text { Healthcare Web } \\
\text { URLs }\end{array}$ & $\begin{array}{l}\text { TF-IDF, Sentiment Polarity, } \\
\text { Grammatical Features }\end{array}$ & $\begin{array}{l}\text { Healthcare web URLs as } \\
\text { True or False }\end{array}$ \\
\hline
\end{tabular}

TABLE II. ReCENT Techniques of Document Similarity in HeAlthCARE Domain AND the Proposed Model TeChNiQues

\begin{tabular}{|l|l|l|l|l|l|}
\hline Sr. No. & Reference & Technique & Dataset & Distance Measure & Similarity \\
\hline 1 & {$[33]$} & RNN, CNN, LR, RNNatt & $\begin{array}{l}59652 \text { discharge summary notes, } \\
344 \text { Wikipedia pages }\end{array}$ & $\begin{array}{l}\text { KSI (Knowledge Source } \\
\text { Integration) }\end{array}$ & Clinical Notes \\
\hline 2 & {$[34]$} & $\begin{array}{l}\text { Classifiers used like SVM, } \\
\text { CRF_based, Rule-Based } \\
\text { and Aggregator }\end{array}$ & $\begin{array}{l}889 \text { records of medication, 1237 of } \\
\text { Obesity, 871 records of VA (each } \\
\text { record is a medical document) }\end{array}$ & Jaccard Distance & Medical Documents \\
\hline 3 & {$[35]$} & K-means & 2673 medical prescriptions & Cosine Similarity & Clinical Notes \\
\hline 4 & Proposed Model & $\begin{array}{l}\text { Classifiers used like LR, } \\
\text { SVM, NB, DT, RF }\end{array}$ & $\mathbf{1 0 0 0}$ Healthcare Web URLs & $\begin{array}{l}\text { Jaccard Distance, } \\
\text { Euclidean Distance, } \\
\text { Cosine Similarity }\end{array}$ & $\begin{array}{l}\text { Fact-Check Healthcare } \\
\text { Web URLs }\end{array}$ \\
\hline
\end{tabular}

\section{Sentiment Analysis and Document Similarity Approaches}

The document classification can be best achieved using document similarity measures. The amalgamation of sentiment analysis and document similarity is effective in terms of document classification as found in the literature. The deep learning techniques along with cosine similarity measures are used to successfully classify documents related to stock news based on the sentiments in literature, resulting in the merging of most relevant documents together [36]. In another approach, One-Class Support Vector Machine (OCSVM) and Latent Semantic Indexing (LSI) were used to classify text documents into positive and negative [37]. In another approach, NETLDA model was proposed to find the semantic similarity between documents using sentiment polarity and cosine similarity approaches [38]. There are three different types of measures followed in the literature for document similarity measurement viz. Jaccard Distance, Cosine Similarity, and Euclidean Distance. However, the authors didn't find any articles with document similarity measures used along with sentiment analysis to classify documents based on their similarity. Thus, the hybrid combination of document similarity and sentiment analysis is a novel approach and can be used to detect and fact-check healthcare related misinformation. Table III displays the recent techniques of document similarity and sentiment analysis and the proposed model techniques

\section{Document Similarity and Fact-Checking}

The major challenge faced in detecting misinformation is performing the fact-checking of data as there fewer benchmark datasets available specific to a certain domain like healthcare. With the enormous amount of information generated online, it is a highly challenging task to perform manual fact-checking of individual articles or blogs available online. Therefore the recent tools and techniques are automated using features from the text like sentimental features, user-specific features, grammatical features, etc. In the literature, authors have used techniques like Term Frequency Inverse Document Frequency (TF-IDF), and cosine similarity measures with k-means, Support Vector Machine, and Multilayer Perceptron to detect credibility of Indonesian news. Also, in another research, Latent Dirichlet Allocation (LDA) and Jaccard distance measures are used to detect fake news on the Buzzfeed dataset. In research to collect evidence for fake news detection word embeddings were used followed by Word Mover's distance measure to measure the similarity between the documents. However, it was observed that Word Mover's distance is very expensive for a large amount of data [39]-[42]. Table IV displays the recent techniques of detecting misinformation using document similarity and sentiment analysis. Though there are few studies handling fact-checking using document similarity measures, not major work is carried out in this field. Thus, in this paper, the authors propose a model with a hybrid combination of sentiment analysis and document similarity approach to detect and fact-check the misinformation. 
TABLE III. ReCEnt TeChNiQues of Document Similarity AND Sentiment ANALysis AND the Proposed Model TeChniQues

\begin{tabular}{|l|l|l|l|l|l|l|}
\hline Sr. No. & Reference & Technique & Dataset & Features & Distance Measure & Application \\
\hline 1 & {$[36]$} & $\begin{array}{l}\text { Deep Neural } \\
\text { Network }\end{array}$ & $\begin{array}{l}62,478 \text { articles related } \\
\text { to stock }\end{array}$ & Sentiment Polarity & Cosine Similarity & $\begin{array}{l}\text { Stock Market News } \\
\text { Similarity Estimation }\end{array}$ \\
\hline 2 & {$[38]$} & NET-LDA & $\begin{array}{l}1518 \text { Turkish reviews } \\
\text { and } 1 \text { K from amazon }\end{array}$ & Sentiment Polarity & $\begin{array}{l}\text { Cosine Similarity } \\
\text { similar documents }\end{array}$ \\
\hline $\mathbf{3}$ & $\begin{array}{l}\text { Proposed } \\
\text { Model }\end{array}$ & $\begin{array}{l}\text { Classifiers used } \\
\text { like LR, SVM, NB, } \\
\text { DT, RF }\end{array}$ & $\begin{array}{l}\mathbf{1 0 0 0} \text { Healthcare Web } \\
\text { URLs }\end{array}$ & $\begin{array}{l}\text { Sentiment Polarity } \\
\text { and Grammatical } \\
\text { Features }\end{array}$ & $\begin{array}{l}\text { Jaccard Distance, } \\
\text { Euclidean Distance, } \\
\text { Cosine Similarity }\end{array}$ & $\begin{array}{l}\text { Detect and Fact-Check } \\
\text { Healthcare Web URLs }\end{array}$ \\
\hline
\end{tabular}

TABLE IV. ReCEnt Techniques of Detecting Misinformation using Document Similarity AND SENTIMENT ANALysis

\begin{tabular}{|l|l|l|l|l|l|l|l|}
\hline Sr. No. & Reference & Technique & Dataset & Features & Distance Measure & Application \\
\hline 1 & {$[39]$} & $\begin{array}{l}\text { K-Means, SVM, } \\
\text { Multilayer } \\
\text { Perceptron }\end{array}$ & $\begin{array}{l}\text { 9038 Fake news } \\
\text { titles \& 1069 Fact } \\
\text { titles }\end{array}$ & Buzzfeed News & TF-IDF & $\begin{array}{l}\text { Credibility } \\
\text { Measurement of } \\
\text { Indonesian News }\end{array}$ \\
\hline 2 & {$[40]$} & Nil & Nil & Self-curated dataset & Word Embeddings & $\begin{array}{l}\text { Word2Vec and Word } \\
\text { Mover's Distance }\end{array}$ & $\begin{array}{l}\text { Evidence Retrieval } \\
\text { for Fake News }\end{array}$ \\
\hline 3 & {$[41]$} & $\begin{array}{l}\text { Proposed } \\
\text { Model }\end{array}$ & $\begin{array}{l}\text { Classifiers used } \\
\text { DT, RF }, \text { RVM, NB, }\end{array}$ & $\begin{array}{l}\text { 1000 Healthcare } \\
\text { Web URLs }\end{array}$ & $\begin{array}{l}\text { Sentiment Polarity } \\
\text { and Grammatical } \\
\text { Features }\end{array}$ & $\begin{array}{l}\text { Jaccard Distance, } \\
\text { Euclidean Distance, } \\
\text { Cosine Similarity }\end{array}$ & $\begin{array}{l}\text { Detect and Fact- } \\
\text { Check Healthcare } \\
\text { Web URLs }\end{array}$ \\
\hline
\end{tabular}

\section{PROPOSED MODEL}

\section{A. Model Architecture}

The proposed model architecture for misinformation detection in the healthcare domain and performing factchecking automatically is shown in Fig. 1. Sections B, C, and $\mathrm{D}$ describe in detail the architecture building. Section B talks about the data collection method, section $\mathrm{C}$ describes the features extracted and used for model building in detail, and section D explains the process of working model.

\section{B. Dataset Creation}

The authors have crawled 60 URLs from the web on the healthcare domain and classified them as True and False with the help of expert opinion. This dataset is used to verify and classify other URLs from the healthcare domain. Further, authors have crawled 898 web URLs related to the healthcare domain. Out of which, 280 URLs are used for training the model and 618 URLs are used for testing purposes. These 1000 URLs are the combination of true and false URLs in the healthcare domain and are classified with the help of document similarity measures, sentimental features, and grammatical features along with machine learning techniques.

\section{Feature Extraction}

There are mainly three different types of features extracted from the URLs datasets. First, the authors focus on sentimental features which include a number of positive word count, number of negative word count, percentage of positive and negative word counts, and the total number of words. In a research to find sentiments of people in a covid-19 pandemic, authors have created a large benchmark dataset based on tweets generated on the twitter [43]. Thus sentimental features are crucial in healthcare domain. In grammatical features, authors have extracted noun, pronoun, verb, and adjectives from the URL text. The third type of feature is document similarity measure. There are three measures used in this paper to factcheck the URLs with manually classified web URLs related to healthcare. The first is Euclidean Distance, which measures the straight line distance between two points in Euclidean space. Equation1 depicts the Pythagorean formula to compute the Euclidean distance between two points $\mathrm{x}$ and $\mathrm{y}$ [44].

$\mathrm{d}(\mathrm{x}, \mathrm{y})=\mathrm{d}(\mathrm{y}, \mathrm{x})$

$=\sqrt{\left(X_{1}-Y_{1}\right)^{2}+\left(X_{2}-Y_{2}\right)^{2}+\cdots+\left(X_{n}-Y_{n}\right)^{2}}$

$\sqrt{\sum_{i=1}^{n}\left(X_{i}-Y_{i}\right)^{2}}$

In this paper, the authors have used Euclidean Distance (ED) measure as a feature computed separately for true and false URLs. The other distance measure used is Jaccard Distance (JD) measures the similarity between two documents by finding the ratio of the size of the intersection and size of the union. Equation2 shows the formula to compute Jaccard Distance between two documents to find the similarity between the documents [44].

$J(A, B)=\frac{|A \cap B|}{|A \cup B|}=\frac{|A \cap B|}{|A|+|B|-|A \cap B|}$

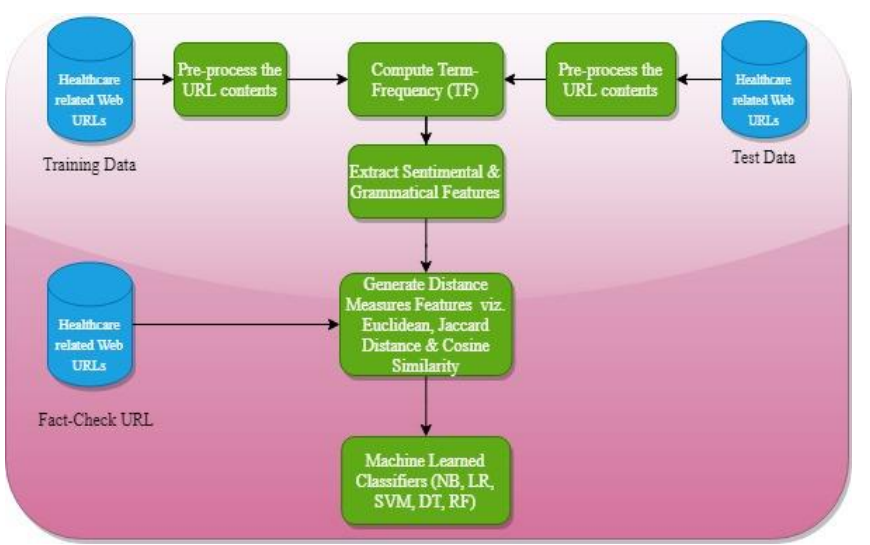

Fig. 1. Proposed Model Architecture for Misinformation Detection and FactChecking. 
Another document similarity measure is the cosine similarity measure. Cosine similarity computes the cosine angle between the vectors. It is represented by the dot product and a magnitude between the vectors. Equation3 shows the formula to compute the cosine similarity between two documents A and B [44].

$\cos (\theta)=\frac{\mathrm{A} \cdot \mathrm{B}}{\|\mathrm{A}\|\|\mathrm{B}\|}$

In this paper, authors have used Euclidean, Jaccard, and Cosine similarity measures as features to perform factchecking of the URLs and thus detect misinformation in the healthcare domain. Table $\mathrm{V}$ lists the final set of features used in the proposed model.

TABLE V. LIST OF ALL THE FEATURES USED IN THE MODEL

\begin{tabular}{|l|l|l|}
\hline Sr. No & Feature Name & Description \\
\hline 1 & Pos_count & Positive count \\
\hline 2 & Neg_count & Negative count \\
\hline 3 & Per_pos_count & Percentage of a positive count \\
\hline 4 & Per_neg_count & Percentage of a negative count \\
\hline 5 & Total_count & Total number of words \\
\hline 6 & Noun & Noun \\
\hline 7 & Pro-noun & Pro-noun \\
\hline 8 & Verb & Verb \\
\hline 9 & Adjective & Adjective \\
\hline 10 & ED_T & Euclidean Distance for True URLs \\
\hline 11 & ED_F & Euclidean Distance for False URLs \\
\hline 12 & JD_T & Jaccard Distance for True URLs \\
\hline 13 & JD_F & Jaccard Distance for False URLs \\
\hline 14 & C_T & Cosine Similarity for True URLs \\
\hline 15 & C_F & Cosine Similarity for False URLs \\
\hline
\end{tabular}

\section{Working Model of Misinformation Detection and Fact-} Checking

In the proposed model, the training dataset is first preprocessed to remove punctuations, stop-words, numeric data, duplicate data, etc. This is required to get the cleaned data for the execution of the model. After pre-processing the URL contents, Term-Frequency (TF) is computed to find the count of terms from the URL textual contents. This term-frequency is stored in the CSV file for future use. The next step is to generate features. The first type of features is sentimental feature that focus mainly on the polarity in terms of positive and negative words of the textual contents from the URL. This is computed to the TF generated in the previous step. Along with sentimental features, grammatical features are also retrieved like noun, pronoun, verb, and adjectives. In misinformation detection, sentimental features play a significant role. It was detected that a text containing misinformation generates more negative words compared to positive words and vice-versa. Thus, more negative sentiments can lead to misinformation [17]. Thus, sentimental features and grammatical features together help to detect misinformation in this proposed model. The next aim is to perform automatic fact-checking of newly arriving URLs from the test dataset. For this reason, a fact-check URL dataset is generated. Fact-
Check URL dataset contains manually fact-checked URLs from healthcare-domain classified into True and False. To perform fact-checking of URLs from the test dataset, the authors have used standard distance measures like Euclidean Distance, Cosine Similarity, and Jaccard Distance as features. Therefore, every URL from the test dataset is first preprocessed to clean the data, term-frequency, and sentimental features are generated and finally, distance measure features are created using the standard formulas explained in section $\mathrm{C}$. To compute the distance measures URL from test dataset is matched with URL from the fact-checked dataset of URLs which gives two numeric values viz. numeric value for distance between true URL from the fact-checked dataset and second numeric value with False URL from the fact-check dataset. These two values are compared and the minimum value is considered as a final feature value. This process is repeated with every URL from the test dataset and for every distance measure. When all the features are generated, machine learning classifiers are applied to test the accuracy of the model. Authors have used five machine learning state-of-theart classifiers from the literature viz. Logistic Regression (LR), Support Vector Machine (SVM), Naïve Bayes (NB), Decision Tree (DT), and Random Forest (RF).

\section{RESULTS AND DISCUSSION}

This section explains the experimental results carried out to evaluate the performance of the model. The proposed methodology is evaluated on five different state-of-the-art classifiers namely LR, SVM, NB, DT, and RF. Section A displays the performance matrix of the model in terms of Accuracy, Precision, Recall, and F1-Score based on the three different parameters viz. Jaccard distance, Euclidean distance, and Cosine similarity distance measures and contains the confusion matrix for the NB classifier. Section B explains the word clouds generated to show the words related to true information and false information from the URLs and Section $\mathrm{C}$ explains the analysis of misinformation detection.

\section{A. Performance Matrix}

The performance matrix is measured in terms of accuracy, precision, recall, and F1-score. Fig. 2 shows the accuracy of the proposed model based on 5 different classifiers. It was observed that NB outperformed all other models in terms of accuracy showing $98.7 \%$ accuracy whereas the decision tree classifier showed less accuracy compared to all other models showing an accuracy of $92.88 \%$.

Fig. 3, Fig. 4 and Fig. 5 display the precision matrix, recall, and F1-score of the proposed model on various classifiers using three parameters viz. Jaccard Distance, Euclidean Distance, and Cosine Similarity measures. Table VI, Table VII and Table VIII display the performance of the distance measure technique used in terms of accuracy, precision, recall, and F1-Score per machine learning classifier. It is observed that the Jaccard Distance Measure showed maximum accuracy compared to other distance measures with maximum accuracy of $98.71 \%$ for the Naïve Bayes classifier whereas the Cosine similarity measure showed minimum accuracy of $88.19 \%$ with the Decision Tree classifier model. Euclidean Distance measure showed average accuracy in comparison with other distance measures. 


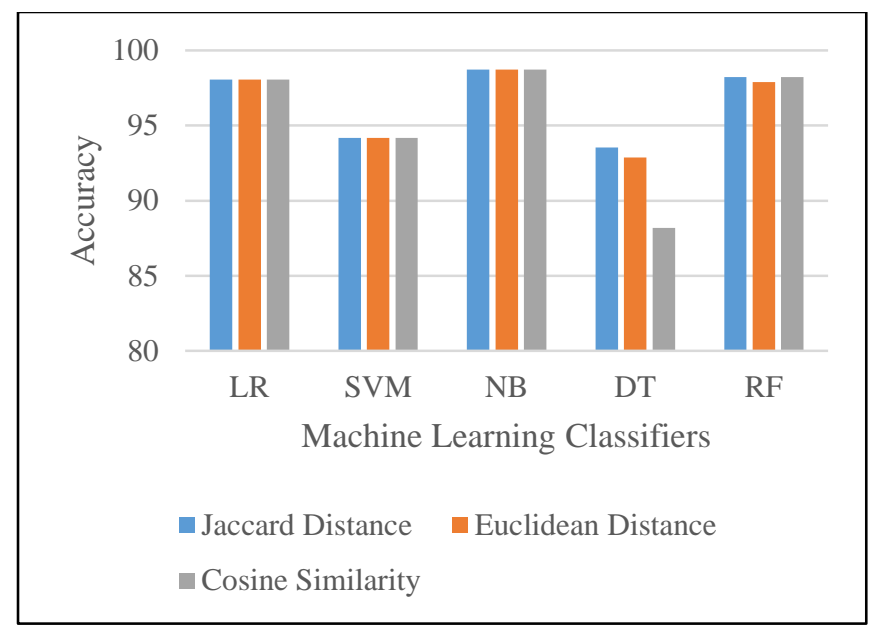

Fig. 2. Performance Matrix in Terms of Accuracy (in Percentage).

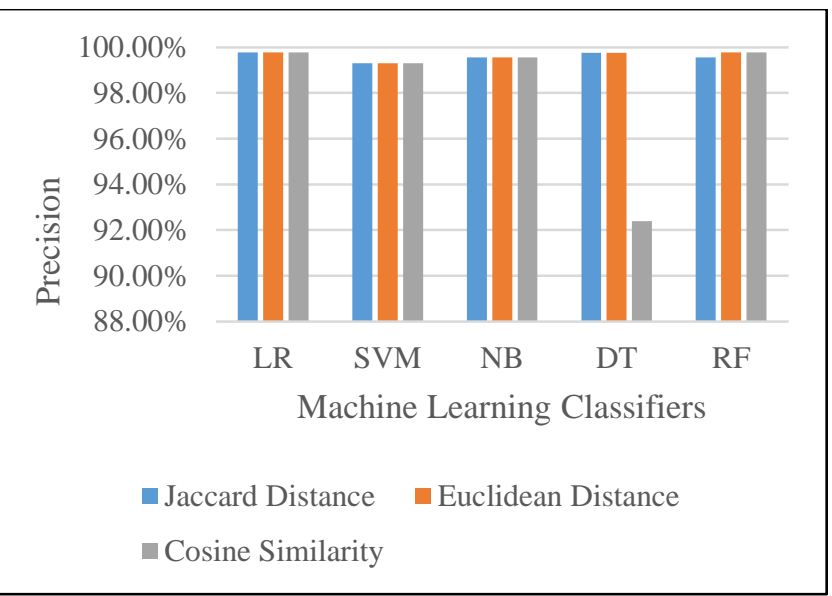

Fig. 3. Performance Matrix in Terms of Precision (in Percentage) of the Proposed Model.

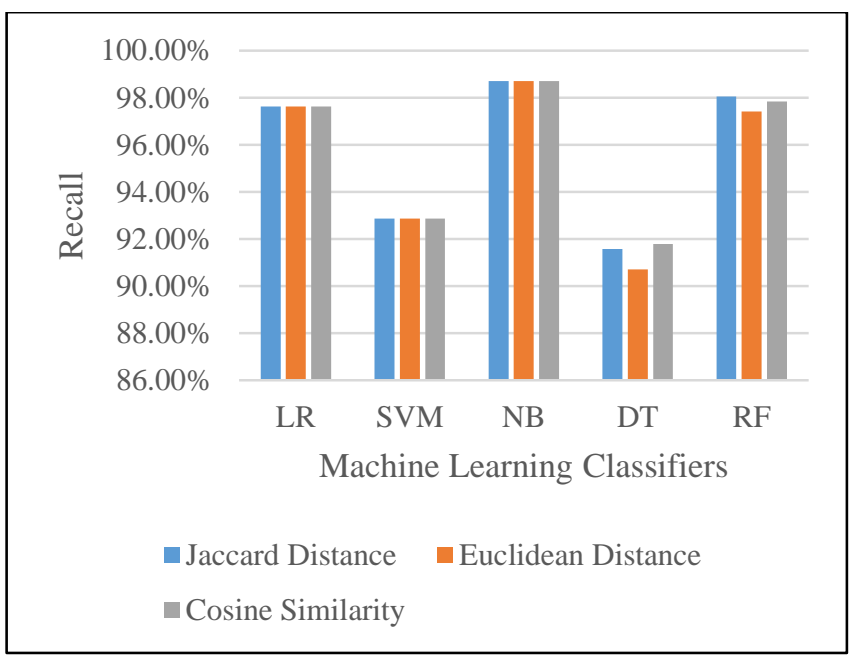

Fig. 4. Performance Matrix in Terms of Recall (in Percentage) of the Proposed Model.

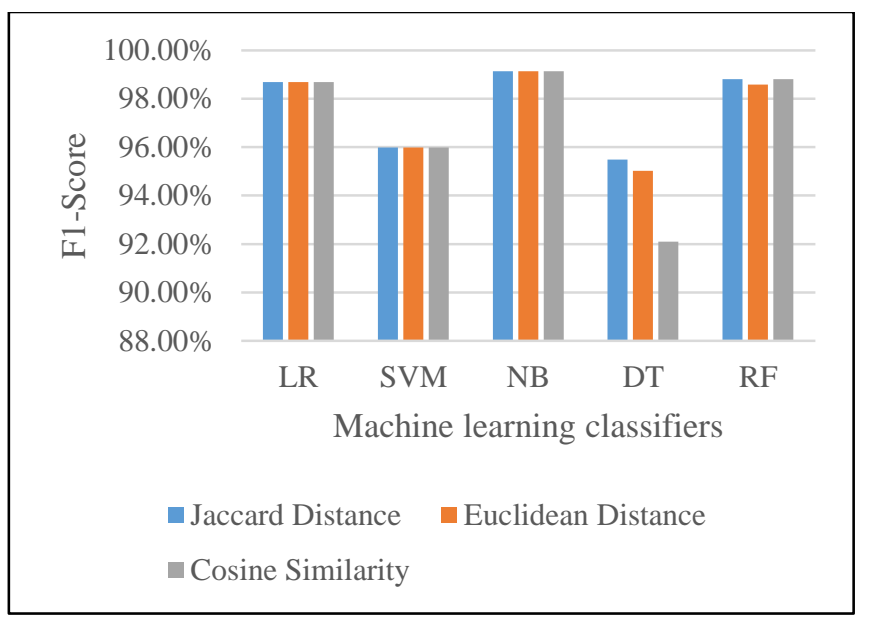

Fig. 5. Performance Matrix in Terms of F1-score (in Percentage) of the Proposed Model.

TABLE VI. Performance Matrix OF JACCARd Distance MeAsure (IN PERCENTAGE) OF THE PROPOSED MODEL

\begin{tabular}{|l|l|l|l|l|}
\hline \multicolumn{5}{|l}{ Jaccard Distance } \\
\hline & Accuracy & Precision & Recall & F1-Score \\
\hline LR & $98.06 \%$ & $99.78 \%$ & $97.62 \%$ & $98.69 \%$ \\
\hline SVM & $94.17 \%$ & $99.31 \%$ & $92.87 \%$ & $95.98 \%$ \\
\hline NB & $98.71 \%$ & $99.56 \%$ & $98.70 \%$ & $99.13 \%$ \\
\hline DT & $93.53 \%$ & $99.76 \%$ & $91.58 \%$ & $95.50 \%$ \\
\hline RF & $98.22 \%$ & $99.56 \%$ & $98.06 \%$ & $98.80 \%$ \\
\hline
\end{tabular}

TABLE VII. PERFormance Matrix of EuClidean Distance MEasure (in Percentage) OF THE PROPOSED Model

\begin{tabular}{|l|l|l|l|l|}
\hline \multicolumn{5}{|l}{ Euclidean Distance } \\
\hline & Accuracy & Precision & Recall & F1-Score \\
\hline LR & $98.06 \%$ & $99.78 \%$ & $97.62 \%$ & $98.69 \%$ \\
\hline SVM & $94.17 \%$ & $99.31 \%$ & $92.87 \%$ & $95.98 \%$ \\
\hline NB & $98.71 \%$ & $99.56 \%$ & $98.70 \%$ & $99.13 \%$ \\
\hline DT & $92.88 \%$ & $99.76 \%$ & $90.71 \%$ & $95.02 \%$ \\
\hline RF & $97.90 \%$ & $99.78 \%$ & $97.41 \%$ & $98.58 \%$ \\
\hline
\end{tabular}

TABLE VIII. Performance Matrix OF Cosine Similarity MEASURE (IN Percentage) OF THE Proposed MODEL

\begin{tabular}{|l|l|l|l|l|}
\hline \multicolumn{5}{|l}{ Cosine Similarity } \\
\hline & Accuracy & Precision & Recall & F1-Score \\
\hline LR & $98.06 \%$ & $99.78 \%$ & $97.62 \%$ & $98.69 \%$ \\
\hline SVM & $94.17 \%$ & $99.31 \%$ & $92.87 \%$ & $95.98 \%$ \\
\hline NB & $98.71 \%$ & $99.56 \%$ & $98.70 \%$ & $99.13 \%$ \\
\hline DT & $88.19 \%$ & $92.39 \%$ & $91.79 \%$ & $92.09 \%$ \\
\hline RF & $98.22 \%$ & $99.78 \%$ & $97.84 \%$ & $98.80 \%$ \\
\hline
\end{tabular}




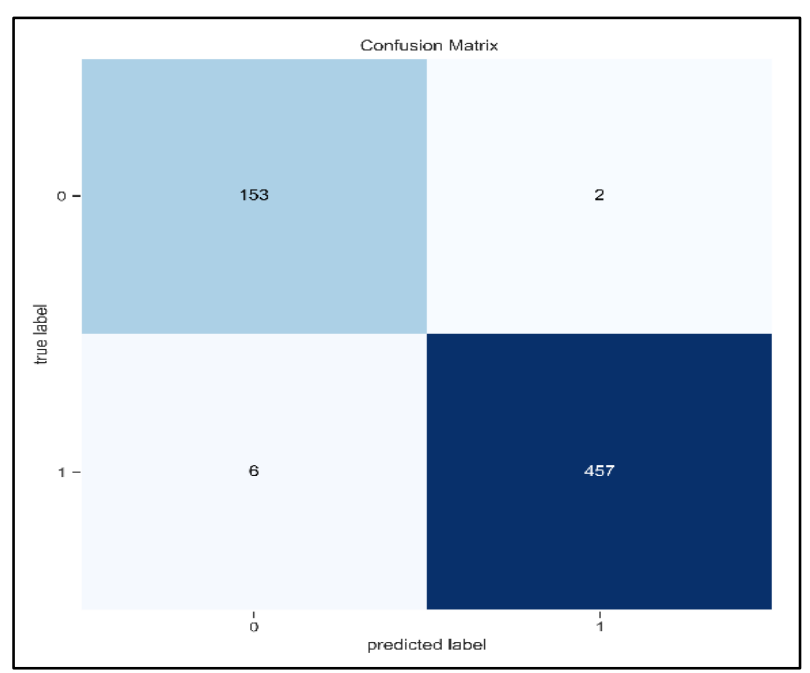

Fig. 6. Confusion Matrix for Naïve Bayes Classifier using Parameters as Euclidean Distance, Jaccard Distance \& Cosine Similarity Measure.

Fig. 6 shows the confusion matrix for Naive Bayes classifier. It can be seen from the confusion matrix that 457 samples are correctly classified as true positive, whereas 153 samples are classified as true negative. False-positive and false-negative sample values are 2 and 6, respectively.

\section{B. Word Clouds}

Fig. 7 and Fig. 8 displays word clouds of URLs having misinformation and legitimate information respectively. It can be seen that the URLs having misinformation contain more negative words like death, false, etc. whereas URLs with true information contain more positive words like well, symptom, increase, etc. This shows that sentiment analysis can play a vital role in detecting misinformation.

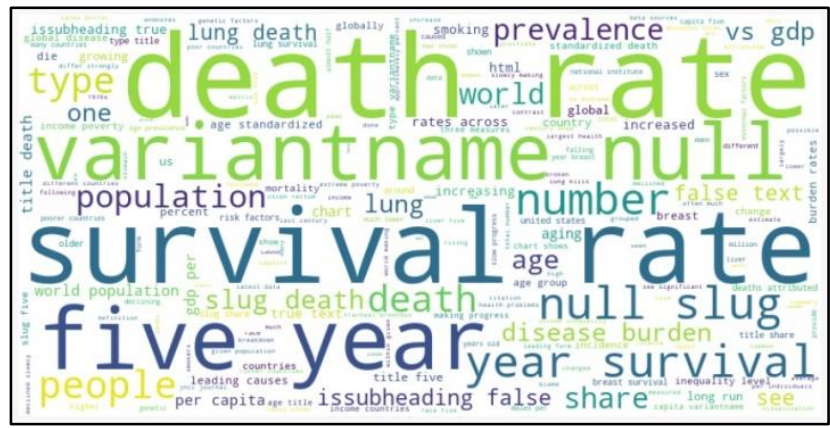

Fig. 7. Word cloud for URLs having Misinformation.

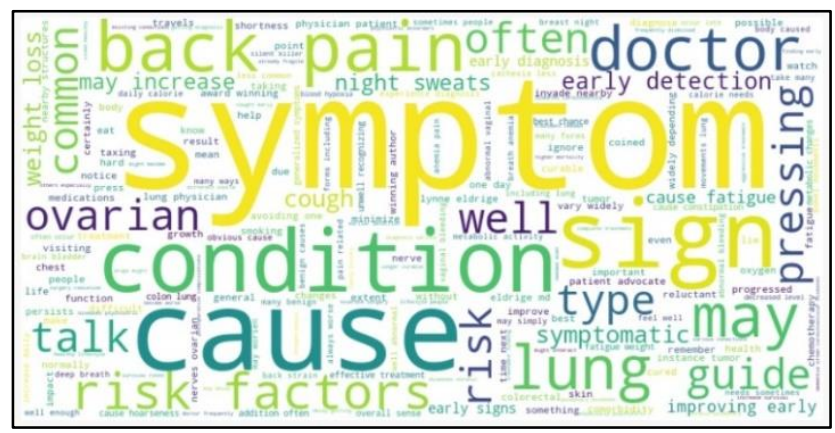

Fig. 8. Word Cloud for URLs having True Information.

\section{Analysis of Misinformation Detected}

Fig. 9 displays the average percentage of misinformation and true information in the web URLs. It can be seen from Fig. 9 that for around 200 URLs the percentage of misinformation is high compared to true information and it is at a peak for URLs ranging from 200 to 300. Fig. 10 displays the average count of positive and negative words in the URLs classified as True. It is been observed that the average positive count of words is $71 \%$ in True URLs and the negative count is $29 \%$. Fig. 11 displays the average count of positive and negative words in the URLs classified as False. It is been observed that the average negative count of words is $62 \%$ in False URLs and the positive count of words is $38 \%$. Thus, the authors found that for URLs with misinformation the average count of negative words is more and positive words are less. Therefore, sentiment analysis is an important feature to detect misinformation in web URLs.

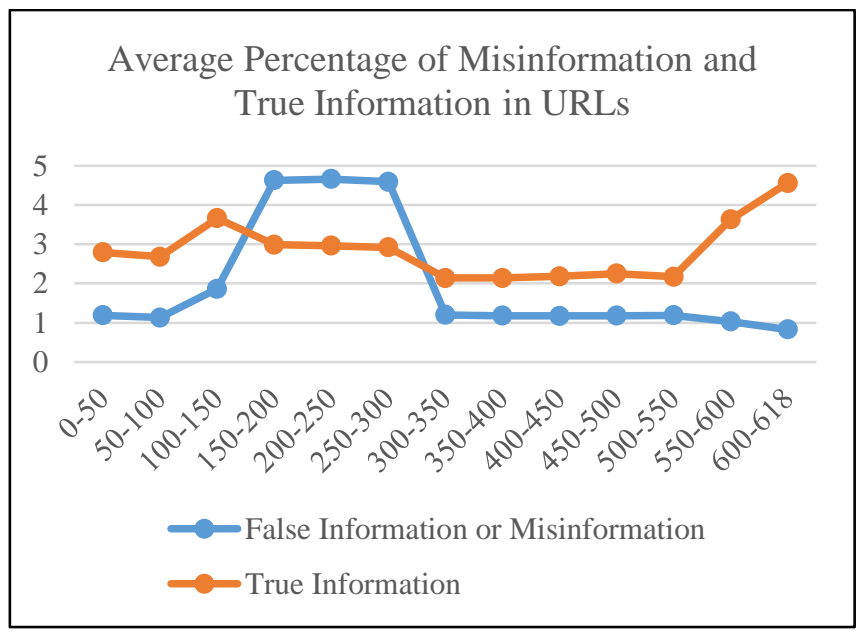

Fig. 9. A Graph showing the Average Percentage of Misinformation and true Information in URLs.

\section{Average percentage of sentiment count in True URLs}

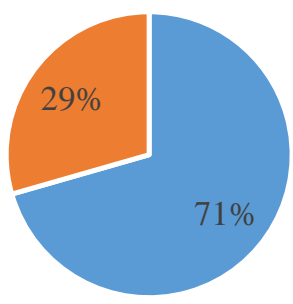

- Positive Count - Negative Count

Fig. 10. A Graph showing the Average Percentage of Positive and Negative Counts in True URLs. 


\section{Average percentage of sentiment count in False URLs}

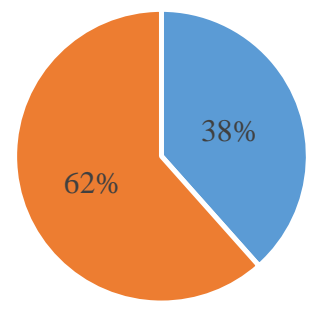

- Positve Word Count - Negative Word Count

Fig. 11. A Graph Showing the Average Percentage of Positive and Negative Counts in False URLs.

\section{CONCLUSION AND FUTURE WORK}

In this research, authors have proposed a model to detect and fact-check misinformation in the healthcare domain. The fact-checking of URLs using distance measures improves the performance of the model than standard techniques of manual fact-checking of data. It was observed that the sentimental features are crucial while detecting misinformation as more negative words is found in URLs containing misinformation compared to the URLs having true information. It was observed that NB outperformed all other models in terms of accuracy showing $98.7 \%$ accuracy whereas the decision tree classifier showed less accuracy compared to all other models showing an accuracy of $92.88 \%$. Also, the Jaccard Distance measure was found to be the best in terms of accuracy compared to Euclidean distance and Cosine similarity measures. In the future, authors want to collect more URLs and observe the difference in the accuracy of the model. Also, the authors want to identify the spreaders of misinformation by keeping track of the percentage of misinformation containing in the text published by these authors.

\section{REFERENCES}

[1] X. Zhou and R. Zafarani, "A Survey of Fake News: Fundamental Theories, Detection Methods, and Opportunities," ACM Comput. Surv., vol. 53, no. 5, 2018, doi: 10.1145/3395046.

[2] K. Shu, D. Mahudeswaran, S. Wang, D. Lee, and H. Liu, "FakeNewsNet: A Data Repository with News Content, Social Context, and Spatiotemporal Information for Studying Fake News on Social Media," Big Data, vol. 8, no. 3, pp. 171-188, 2020, doi: 10.1089/big.2020.0062.

[3] C. Carvalho, N. Klagge, and E. Moench, "The persistent effects of a false news shock," J. Empir. Financ., vol. 18, no. 4, pp. 597-615, 2011, doi: 10.1016/j.jempfin.2011.03.003.

[4] S. Lewandowsky, U. K. H. Ecker, C. M. Seifert, N. Schwarz, and J. Cook, "Misinformation and Its Correction: Continued Influence and Successful Debiasing," Psychol. Sci. Public Interes. Suppl., vol. 13, no. 3, pp. 106-131, 2012, doi: 10.1177/1529100612451018.

[5] Y. Zhao, J. Da, and J. Yan, "Detecting health misinformation in online health communities: Incorporating behavioral features into machine learning based approaches," Inf. Process. Manag., vol. 58, no. 1, 2021, doi: 10.1016/j.ipm.2020.102390.

[6] P. Meel and D. K. Vishwakarma, "Fake news, rumor, information pollution in social media and web: A contemporary survey of state-ofthe-arts, challenges and opportunities," Expert Syst. Appl., vol. 153, 2020, doi: 10.1016/j.eswa.2019.112986.
[7] Y. Wang, M. McKee, A. Torbica, and D. Stuckler, "Systematic Literature Review on the Spread of Health-related Misinformation on Social Media," Soc. Sci. Med., vol. 240, 2019, doi: 10.1016/j.socscimed.2019.112552.

[8] I. Secosan, D. Virga, Z. P. Crainiceanu, L. M. Bratu, and T. Bratu, "Infodemia: Another enemy for romanian frontline healthcare workers to fight during the covid-19 outbreak," Med., vol. 56, no. 12, pp. 1-9, 2020, doi: 10.3390/medicina56120679.

[9] S. Tejedor, A. Pérez-Escoda, A. Ventín, F. Tusa, and F. Martínez, "Tracking websites' digital communication strategies in latin american hospitals during the COVID-19 pandemic," Int. J. Environ. Res. Public Health, vol. 17, no. 23, pp. 1-19, 2020, doi: 10.3390/ijerph17239145.

[10] A. P. Worrall et al., "Readability of online COVID-19 health information: a comparison between four English speaking countries," BMC Public Health, vol. 20, no. 1, 2020, doi: 10.1186/s12889-020-09710-5.

[11] A. Habib, M. Z. Asghar, A. Khan, A. Habib, and A. Khan, "False information detection in online content and its role in decision making: a systematic literature review," Soc. Netw. Anal. Min., vol. 9, no. 1, 2019, doi: 10.1007/s13278-019-0595-5.

[12] P. Kaur, R. S. Boparai, and D. Singh, "Hybrid text classification method for fake news detection," Int. J. Eng. Adv. Technol., vol. 8, no. 5, pp. 2388-2392, 2019, [Online]. Available: https://www.scopus.com/inward/record.uri?eid=2-s2.085069445671\&partnerID=40\&md5=67b1d4b3b93f226a348b7859e1eaeff 6.

[13] F. A. Ozbay and B. Alatas, "Fake news detection within online social media using supervised artificial intelligence algorithms," Phys. A Stat. Mech. its Appl., vol. 540, p. 123174, 2020.

[14] J. I. De Morais, H. Q. Abonizio, G. M. Tavares, A. A. Da Fonseca, and S. Barbon, "Deciding among fake, satirical, objective and legitimate news: A multi-label classification system," in ACM International Conference Proceeding Series, 2019, doi: 10.1145/3330204.3330231.

[15] K. Popat, S. Mukherjee, J. Strötgen, and G. Weikum, "Credibility assessment of textual claims on the web," in International Conference on Information and Knowledge Management, Proceedings, 2016, vol. 24-28Octo, pp. 2173-2178, doi: 10.1145/2983323.2983661.

[16] C. S. de Britto Almeida and D. A. Santos, "Text similarity using word embeddings to classify misinformation," in CEUR Workshop Proceedings, 2020, vol. 2607, pp. 63-68, [Online]. Available: https://www.scopus.com/inward/record.uri?eid=2-s2.085085651452\&partnerID=40\&md5=dfe87346ce88014b2c2fe83b25bd17 37.

[17] Z. Xu and H. Guo, "Using Text Mining to Compare Online Pro- and Anti-Vaccine Headlines: Word Usage, Sentiments, and Online Popularity," Commun. Stud., vol. 69, no. 1, pp. 103-122, 2018, doi: 10.1080/10510974.2017.1414068.

[18] A. Benamira, B. Devillers, E. Lesot, A. K. Ray, M. Saadi, and F. D. Malliaros, "Semi-supervised learning and graph neural networks for fake news detection," in Proceedings of the 2019 IEEE/ACM International Conference on Advances in Social Networks Analysis and Mining, ASONAM 2019, 2019, pp. 568-569, doi: 10.1145/3341161.3342958.

[19] E.-S. M. El-Alfy and S. Al-Azani, "Statistical comparison of opinion spam detectors in social media with imbalanced datasets," Commun. Comput. Inf. Sci., vol. 969, pp. 157-167, 2019, doi: 10.1007/978-981-135826-5_12.

[20] I. Ahmad, M. Yousaf, S. Yousaf, and M. O. Ahmad, "Fake News Detection Using Machine Learning Ensemble Methods," Complexity, vol. 2020, 2020, doi: 10.1155/2020/8885861.

[21] G. L. Ciampaglia, P. Shiralkar, L. M. Rocha, J. Bollen, F. Menczer, and A. Flammini, "Computational fact checking from knowledge networks," PLoS One, vol. 10, no. 6, 2015, doi: 10.1371/journal.pone.0128193.

[22] I. Agarwal and D. P. Rana, "Credibility of misinformation and the science of sentiments," J. Adv. Res. Dyn. Control Syst., vol. 12, no. 7 Special Issue, pp. 1738-1745, 2020, doi: 10.5373/JARDCS/V12SP7/20202283.

[23] M. A. Alonso, D. Vilares, C. Gómez-Rodríguez, and J. Vilares, "Sentiment analysis for fake news detection," Electron., vol. 10, no. 11, 2021, doi: 10.3390/electronics10111348.

[24] R. Asha, R. Jain, G. Das, and P. Bharadwaj, "Sentimental analysis and detection of rumours for social media data using logistic regression," Int. 
J. Innov. Technol. Explor. Eng., vol. 9, no. 1, pp. 2123-2126, 2019, doi: 10.35940/ijitee.A4670.119119.

[25] B. R. Prathap, A. K. Sujatha, C. B. S. Yadav, and M. Mounika, "Polarity detection on real-time news data using opinion mining," Adv. Parallel Comput., vol. 37, pp. 90-97, 2020, doi: 10.3233/APC200124.

[26] V. Raghupathi, J. Ren, and W. Raghupathi, "Studying public perception about vaccination: A sentiment analysis of tweets," Int. J. Environ. Res. Public Health, vol. 17, no. 10, 2020, doi: 10.3390/ijerph17103464.

[27] Y. Yang, L. Zheng, J. Zhang, Q. Cui, Z. Li, and P. S. Yu, "TI-CNN: Convolutional Neural Networks for Fake News Detection," 2018, [Online]. Available: http://arxiv.org/abs/1806.00749.

[28] C. Castillo, M. Mendoza, and B. Poblete, "Information credibility on Twitter," Proc. 20th Int. Conf. Companion World Wide Web, WWW 2011, no. January, pp. 675-684, 2011, doi: 10.1145/1963405.1963500.

[29] D. C. Edara, L. P. Vanukuri, V. Sistla, and V. K. K. Kolli, "Sentiment analysis and text categorization of cancer medical records with LSTM," J. Ambient Intell. Humaniz. Comput., 2019, doi: 10.1007/s12652-01901399-8.

[30] P. M. Arunkumar, S. Chandramathi, and S. Kannimuthu, "Sentiment analysis-based framework for assessing internet telemedicine videos," Int. J. Data Anal. Tech. Strateg., vol. 11, no. 4, pp. 328-336, 2019, doi: 10.1504/IJDATS.2019.103755.

[31] A. Yadav and D. K. Vishwakarma, "A Weighted Text Representation framework for Sentiment Analysis of Medical Drug Reviews," in Proceedings - 2020 IEEE 6th International Conference on Multimedia Big Data, BigMM 2020, 2020, pp. 326-332, doi: 10.1109/BigMM50055.2020.00057.

[32] W. M. S. Yafooz and A. Alsaeedi, "Sentimental Analysis on HealthRelated Information with Improving Model Performance using Machine Learning," J. Comput. Sci., vol. 17, no. 2, pp. 112-122, 2021, doi: 10.3844/jcssp.2021.112.122.

[33] T. Bai and S. Vucetic, "Improving medical code prediction from clinical text via incorporating online knowledge sources," in The Web Conference 2019 - Proceedings of the World Wide Web Conference, WWW 2019, 2019, pp. 72-82, doi: 10.1145/3308558.3313485.

[34] X.-B. Li and J. Qin, "Anonymizing and sharing medical text records," Inf. Syst. Res., vol. 28, no. 2, pp. 332-352, 2017, doi: 10.1287/isre.2016.0676.
[35] E. Naaz, D. Sharma, D. Sirisha, and M. Venkatesan, "Enhanced K-means clustering approach for health care analysis using clinical documents," Int. J. Pharm. Clin. Res., vol. 8, no. 1, pp. 60-64, 2016, [Online]. Available: $\quad$ https://www.scopus.com/inward/record.uri?eid=2-s2.084965097226\&partnerID $=40 \& \mathrm{md} 5=\mathrm{e} 406 \mathrm{~b} 59 \mathrm{~b} 8 \mathrm{e} 4787 \mathrm{~d} 8 \mathrm{~d} 0 \mathrm{c} 5083 \mathrm{a} 31862 \mathrm{c}$ 2a.

[36] H. Yanagimoto, M. Shimada, and A. Yoshimura, "Document similarity estimation for sentiment analysis using neural network," in 2013 IEEE/ACIS 12th International Conference on Computer and Information Science, ICIS 2013 - Proceedings, 2013, pp. 105-110, doi: 10.1109/ICIS.2013.6607825.

[37] B. Shravan Kumar and V. Ravi, "One-class text document classification with OCSVM and LSI," Adv. Intell. Syst. Comput., vol. 517, pp. 597606, 2017, doi: 10.1007/978-981-10-3174-8_50.

[38] E. Ekinci and S. I. OMURCA, "NET-LDA: A novel topic modeling method based on semantic document similarity," Turkish J. Electr. Eng. Comput. Sci., vol. 28, no. 4, pp. 2244-2260, 2020, doi: 10.3906/ELK1912-62.

[39] R. Arianto, S. Warnars, H. Leslie, and Y. Heryadi, "FAKE NEWS DETECTION MODEL BASED ON CREDIBILITY MEASUREMENT FOR INDONESIAN ONLINE,” vol. 99, no. 7, pp. 1571-1593, 2021.

[40] K. Xu, F. Wang, H. Wang, and B. Yang, "Detecting fake news over online social media via domain reputations and content understanding," Tsinghua Sci. Technol., vol. 25, no. 1, pp. 20-27, 2020, doi: 10.26599/TST.2018.9010139.

[41] H. Gong, T. Sakakini, S. Bhat, and J. Xiong, "Document similarity for texts of varying lengths via hidden topics," ACL 2018 - 56th Annu. Meet. Assoc. Comput. Linguist. Proc. Conf. (Long Pap., vol. 1, pp. 2341-2351, 2018, doi: 10.18653/v1/p18-1218.

[42] V. Gupta, K. Beckh, S. Giesselbach, and D. Wegener, "Supporting verification of news articles with automated search for semantically similar articles," pp. 1-13, 2021.

[43] U. Naseem, I. Razzak, M. Khushi, P. W. Eklund, and J. Kim, "COVIDSenti: A Large-Scale Benchmark Twitter Data Set for COVID19 Sentiment Analysis," IEEE Trans. Comput. Soc. Syst., vol. 8, no. 4, pp. 976-988, 2021, doi: 10.1109/TCSS.2021.3051189.

[44] P. Marjai, P. Lehotay-Kéry, and A. Kiss, "Document similarity for error prediction," J. Inf. Telecommun., 2021, doi: $10.1080 / 24751839.2021 .1893496$. 